\title{
A Notional Duette on Nanophotonics Fundamentals
}

\section{Emmanuel A. Anagnostakis}

Hellenic Physical Society, Alimos, Greece

Email:emmanagn@otenet.gr

How to cite this paper: Anagnostakis, E.A (2018) A Notional Duette on Nanophotonics Fundamentals. Optics and Photonics Journal, 8, 317-322.

https://doi.org/10.4236/opj.2018.811027

Received: August 2, 2018

Accepted: November 17, 2018

Published: November 20, 2018

Copyright $\odot 2018$ by author and Scientific Research Publishing Inc. This work is licensed under the Creative Commons Attribution International License (CC BY 4.0).

http://creativecommons.org/licenses/by/4.0/

\section{c) (i) Open Access}

\begin{abstract}
A two-part Notional Synthesis on Nanophotonics Fundamentals is being carried out: On the one hand, a rather novel depiction of the Fermionic Quantum Causality is being attempted. On the other hand, a Nanophotonic Response Encoder is being devised: Illuminated Electrons are the original Protagonists.
\end{abstract}

\section{Keywords}

Nanophotonics, Fermions, Nanodevice Photoresponse

\section{Primo: On Fermionic Quantum Causality}

With respect to the Causality of the Fermi-Dirac Distribution Function

$$
f(x)=\frac{1}{\mathrm{e}^{x}+1}
$$

it can be easily observed that, since

$$
f^{\prime}(x)=-\frac{\mathrm{e}^{x}}{\left(\mathrm{e}^{x}+1\right)^{2}}
$$

and

$$
f(x)-1=-\frac{\mathrm{e}^{x}}{\mathrm{e}^{x}+1}
$$

there holds that

$$
f^{\prime}=f(f-1)=f^{2}-f
$$

under the Initial Condition

$$
f(0)=\frac{1}{2}
$$

a Second-Order Constant-Coefficient Bernoulli Differential Equation (DE) emerg- 
ing as embodying the (Quantum) Causality of $f$.

Indeed, by defining

$$
\varphi(x)=\frac{1}{f(x)}
$$

$(f(x)$ being positive for any Real $x$, and only asymptotically tending to zero for $X$ tending to plus infinity) there appears the Conjugate First-Order ConstantCoefficient Linear Non-Homogeneous DE

$$
\varphi^{\prime}=\varphi-1
$$

being subject to the Initial Condition

$$
\varphi(0)=2
$$

which is satisfied by the sum of its (unconstrained) Partial Solution $\varphi_{p}=1$ and the Solution of its Respective Homogeneous DE (constrained through (8)) $\varphi_{H}=$ $\exp x$.

$$
\varphi(x)=\mathrm{e}^{x}+1
$$

leading to (1) through (6).

Easily verifiably, on the other hand, the Monoparametric Family of Constant-Coefficient Complete Riccati DEs

$$
y^{\prime}=y^{2}-(2 \rho+1) y+\rho(\rho+1)
$$

$\rho$ being an arbitrarily chosen Real Number different from both $(-1)$ and 0 , might be regarded as a Collective Generator of the Fermi - Dirac Distribution Function Causality (4) by virtue of the simple Transformation

$$
y(x)=f(x)+\rho
$$

leading back to (4) for any such $\rho$.

(An Adjoint Mathematical Treatment concerning the Bose -Einstein Distribution Function is, obviously, possible; the respective overall Notional Interpretation would need to be crucially distinct.)

Returning, now, to the Bernoulli DE (4) plausibly acceptable for alternatively summarising a Quantal Causality of the distribution of a Fermionic Ensemble within its valid Zone of accessible Energy Levels one may interpret the RHS as factorising the (Effective or Reduced) Energy Rate of Change $\mathrm{d} f(x) / \mathrm{d} x$ of the Level-Occupation Probability $f(x), x$ denoting $\left(E-E_{F}\right) /(k T)$, with $E_{F}$ being the pertinent Fermi Level of the Thermodynamic Ensemble (consistent with the Initial Condition (5)), E referring to the In-Zone Energy Position of "Current" Level, and $k T$ being the indicative Fermionic Thermal Energy, into the Product of $f(x)$ itself and the Opposite of its Complementary Probability $(1-f(x))$, being equivalently the probability of Non-Occupancy of (Allowed Energy States of) Energy Level $E=x \cdot k T+E_{F}$.

Given, furthermore, that by virtue of Ergodicity the Occupation Probability of an Energy Level connotes the Percentage of Occupied States (over the Respective Set of Allowed States) of the Energy Level, the Causality potentially implied by 
the above factorisation appears even more comprehensible: The Reduced Energy Rate at which $f(x)$ is changing (actually decreasing, $f^{\prime}=f(f-1)=-f(1-f)$, with Probability $f$ lying between 0 and 1 for any bound Real $x$ ) is overlappingly analogous both to the Occupancy Degree of Current Energy Level and (in parallel) to the Non-Occupancy Degree of Current Energy Level.

Such a (Simulative) Quantum Specific Equipotently Dual Proportionality of $f^{\prime}$ to $f$ and $(1-f)$, the Non-Linearity of DE (4) not being overlooked and the brilliance of systematic Topical Research Studies (for example, Refs [1] [2] [3]) being enthusiastically acknowledged, could be ventured to be considered linked to the Pauli-Principle-respecting Singularity of the Fermionic Character.

As Dirac, Fermi, Einstein, Feynman, and their Peers, would comment upon, Nature favours (Inherent) Functional Symmetries, the echo of some of which may be appearing perceptible through (Phenomenological) Physical Relationships of Admirably Concise Purity.

\section{Secondo: On a Nanophotonic Response Encoder}

In previous studies of ours, there has been traced an approximate analogy between the Photonic Dose $\beta$ Rate of Change $(\mathrm{d} \eta / \mathrm{d} \beta)$ of the Persistent Photocarrier Sheet Density $\eta$ and the Average Conductivity Carrier Mobility $\mu$ [4], allowing for the expression of the Second Photonic Dose Derivative $\eta_{\beta \beta}=\mathrm{d}^{2} \eta / \mathrm{d} \beta^{2}$ of the Surface Concentration $\eta$ as a Nanodevice-specific multiple of the Photonic Dose Rate of Change $\mu_{\beta}=\mathrm{d} \mu / \mathrm{d} \beta$ of Carrier Mobility $\mu$ :

$$
\eta_{\beta \beta}+M \mu_{\beta}=0, M<0
$$

Equation (12) already incorporates the experimentally monitored Limiting Linearity of $\eta(\beta)$ for approaching the saturation of the Nanophotonic Device Fundamental Conduction Subband, signaled by the instantaneous vanishing of $\mu_{\beta}$.

The physically meaningful Boundary Conditions reflect that the Photoinduced Electron Surface Concentration scans the scale from its naught Dark-Value to its Terminal Value $\eta_{0}$ compatible with the Capacity of the eventually (at Critical Total Photonic Dose $\beta_{0}$ ) saturated Fundamental Subband

$$
\eta(0)=0 \quad \& \quad \eta\left(\beta_{0}\right)=\eta_{0}
$$

Mathematically, the Solution of Equation (12) under the Inhomogeneous Boundary Conditions (13) is formulated as the Superposition of a function $\chi(\beta)$ satisfying the respective Homogeneous DE

$$
\chi_{\beta \beta}=0
$$

subject to the Inhomogeneous Terminal Condition

$$
\chi(0)=0 \quad \& \quad \chi\left(\beta_{0}\right)=\eta_{0}
$$

and a function $\psi(\beta)$ verifying the Complete, Inhomogeneous

$$
\psi_{\beta \beta}+M \mu_{\beta}=0
$$

under the associated Homogeneous Terminal Conditions 


$$
\psi(0)=0 \& \psi\left(\beta_{0}\right)=0
$$

Obviously, the Particular Solution of Equation (14) consistent with the Requirement (15) is

$$
\chi(\beta)=\eta_{0} \frac{\beta}{\beta_{0}}
$$

On the other hand, for the Complete Equation (16) holding under Conditions (17) there may be employed the respective Green's Function $g(\beta, \gamma)$, which for a reference Cumulative Photonic Dose $\gamma$ reads:

$$
g(\beta, \gamma)=-\frac{B(\beta) \Gamma(\gamma)}{w[B, \Gamma]_{\gamma}} \text { for } \beta<\gamma
$$

$\&$

$$
g(\beta, \gamma)=-\frac{B(\gamma) \Gamma(\beta)}{w[B, \Gamma]_{\gamma}} \text { for } \beta>\gamma
$$

$B(\beta)$ being the Solution of the Homogeneous Equation

$$
B_{\beta \beta}=0
$$

under the Condition

$$
B(0)=0
$$

and $\Gamma(\gamma)$ being the Solution of the Homogeneous Equation

$$
\Gamma_{\beta \beta}=0
$$

under the Condition

$$
\Gamma\left(\beta_{0}\right)=0
$$

and $w[B, \Gamma]_{\gamma}$ being the Wronskian Determinant of functions $B$ and $\Gamma$ evaluated at the reference Instantaneous Cumulative Photonic Dose $\gamma$.

Thus, the Green's Function takes the form

$$
g(\beta, \gamma)=\left(\beta_{0}-\gamma\right) \frac{\beta}{\beta_{0}} \text { for } \beta<\gamma
$$

$\&$

$$
g(\beta, \gamma)=\left(\beta_{0}-\beta\right) \frac{\gamma}{\beta_{0}} \text { for } \beta>\gamma
$$

Upon which the solution of Equation (16) consistent with Conditions (17) is derivable as the Convolution between the Green's Function g and the Stimulus $M \mu_{\beta}$ :

$$
\psi(\beta)=\int_{0}^{\beta_{0}} g(\beta, \gamma) M \mu_{\beta}(\gamma) \mathrm{d} \gamma
$$

In the light of the fact that the Green's Function is Nanodevice-specific as parametrised by the characteristic Total Photonic Dose $\beta_{0}$ tantamount to the saturation of the Capacity of its Fundamental Conduction Subband, it appears meaningful to adopt $g(\beta, \gamma)$ as a potentially Notionally Universal Nanophotonic Re- 
sponse Encoder (NRE).

Owing to the symmetry of the NRE with respect to interchanging its two Arguments $\beta$ and $\gamma, \psi(\beta)$ being generated by the Convolution between the Continuous, Real-valued, Symmetric, double-Argument function $g(\beta, \gamma)$ and the Continuous, Real-valued, single-Argument function $M \mu_{\beta}^{\prime}(\beta)$, determined by the Nanophotonic nature of the each-time experimental Device and its measured $\mu_{\beta}^{\prime}(\beta)$ Photoresponse, can be represented by a Linear Combination (through a Series $\kappa_{v}$ of pertinent Weighting Coefficients) of the Eigenfunctions $\psi_{v}(\beta)$ of the Homogeneous Fredholm Integral Equation having the particular double-Argument function $g(\beta, \gamma)$ as its Kernel

$$
\psi(\beta)=\sum_{v}\left\{\kappa_{v} \psi_{v}(\beta)\right\}
$$

with $\psi_{v}(\beta)$ being the $v$-th order Eigenfunction of the Homogeneous Fredholm (Convolution) Integral Equation

$$
\psi_{v}(\beta)=\alpha_{v} \int_{0}^{\beta_{0}} g(\beta, \gamma) \psi_{v}(\gamma) \mathrm{d} \gamma
$$

with $\alpha_{v}$ denoting the respective $v$-th order Real Eigenvalue (fulfilling the property that to each pair of Distinct Eigenvalues there corresponds a pair of mutually Orthogonal Eigenfunctions), and the NRE embodying the Fredholm Kernel.

Therefore, $\psi(\beta)$ is derived through Equation (28) as the Resultant of the Orthogonal Component Eigenfunctions $\psi_{\nu}(\beta)$ scaled by the appropriate Real Coefficients $\kappa_{v}$ determined through the Photoenhancement-associated Stimulus Function $M \mu_{\beta}(\beta)$ (simulatively) energising the Nanophotonic Response of the eachtime experimentally monitored Semiconductor Fermionic Device.

\section{Conclusions}

A Notional Duette on Nanophotonics Fundamentals has been carried out: On the one hand, a Scheme of Quantum Specific Equipotently Dual Proportionality of the Reduced Energy Rate of Change $f$ of the Fermi-Dirac Function $f$ to $f$ itself and to its Complementary Probability $(1-f)$ has been perceived as plausibly connoting a functional Fermionic Quantal Causality.

On the other hand, a Notionally Universal Nanophotonic Response Encoder has been adopted in terms of the Kernel of the Fredholm Integral Equation providing the Orthogonal Spectrum attached to the Limiting Behaviour for causally picturing the Complete Nanophotonic Response Function of a typical Semiconductor Heterointerface Device.

\section{Conflicts of Interest}

The author declares no conflicts of interest regarding the publication of this paper.

\section{References}

[1] Fukushima, T. (2015) Precise and Fast Computation of Fermi-Dirac Integral of In- 
teger and Half-Integer Order by Piece-Wise Mini-Max Rational Approximation. Applied Mathematics and Computation, 259, 708-729.

https://doi.org/10.1016/j.amc.2015.03.009

[2] Schuch, D. (2014) Non-Linear Riccati Equations as a Unifying Link between Linear Quantum Mechanics and Other Fields of Physics. Journal of Physics. Conference Series, 504, 012005. https://doi.org/10.1088/1742-6596/504/1/012005

[3] Chaudhry, M.A., et al. (2010) Extended Fermi-Dirac and Bose-Einstein Functions with Applications to the Family of zeta Functions. Cornell University Library, Arxiv: $1004.0588 \mathrm{vl}$ math-ph.

[4] Anagnostakis, E.A. (2011) On Negative Differential Mobility in Nanophotonic Device Functionality. Optics and Photonics Journal, 1, 216-220.

https://doi.org/10.4236/opj.2011.14033 